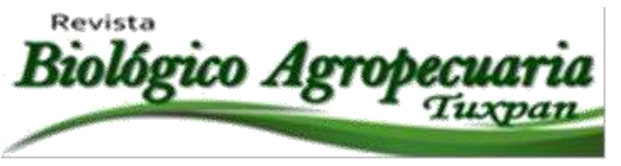

\title{
Efectividad biológica del nematicida oxamyl en el control de Meloidogyne spp. en
} tomate Solanum lycopersicum

Biological effectiveness of oxamyl nematicide in the control of Meloidogyne spp. in tomato Solanum lycopersicum

Figueroa Hernández Víctor Manuel ${ }^{1 \bowtie}$, Hernández Mar Brenda Mariana ${ }^{1}$ y Román Martínez Mario Alberto $^{1}$

${ }^{1}$ Facultad de Ciencias Biológicas y Agropecuarias de la Universidad Veracruzana. Campus Tuxpan

${ }^{凶}$ Autor para correspondencia: vfigueroa@uv.mx

Recibido: $23 / 08 / 2016$

Aceptado: 24/11/2016

\section{RESUMEN}

El nemátodo agallador de raíces Meloidogyne spp., es una plaga de gran importancia económica en todo el mundo; se reportan pérdidas de la producción mundial sobre todo en productos horto-frutícolas por causa de este nemátodo en aproximadamente 115 mil millones de dólares anuales por lo general los productores no detectan el problema antes de la siembra; el estudio busca la efectividad biológica de un nematicida a diferentes dosis para el control de Meloidogyne spp. en tomate. El experimento se estableció en el municipio de Tamiahua, Veracruz, usando un diseño de bloques completos al azar, con 5 tratamientos y 4 repeticiones, evaluando los siguientes productos: Nematicida Vydate L® a dosis de $0.75 \mathrm{lts} / \mathrm{ha}^{-1}, 1.0 \mathrm{lts} / \mathrm{ha}^{-1}$ y $2.0 \mathrm{lts} / \mathrm{ha}^{-1}$, el nematicida Nemator ${ }^{\circledR}$ a dosis de 1.0 $\mathrm{kg} / \mathrm{ha}^{-1}$, y un testigo absoluto; realizando 3 aplicaciones al cultivo, estas se llevaron a cabo al momento del trasplante, a los 20 días después del trasplante (ddt), a los 40 y 60 ddt, respectivamente. El Nematicida Nemator ${ }^{\circledR}$ a dosis de $1.0 \mathrm{~kg} / \mathrm{ha}^{-1}$ controla satisfactoriamente el agallamiento en tomate causado por Meloidogyne spp. al igual que Vydate $\mathrm{L} \circledast$ a dosis de $2.0 \mathrm{lts} / \mathrm{ha}^{-1}$, además este último, presenta buen control contra insectos chupadores, en el testigo se encontró presencia de pulgón Myzus persicae, comparado con el resto de los tratamientos. En base al análisis de los resultados, se recomienda tratamiento en plántula debido a que mejora la asimilación y existe ahorro de productos.

Palabras clave: Nemátodo, control, tomate. 


\begin{abstract}
The root-knot nematode Meloidogyne spp, is a pest of great economic importance throughout the world; world production losses especially in Horto-fruit products are reported because of this nematode in about 115 billion dollars annually producers usually do not detect the problem before planting; the study seeks biological effectiveness of a nematicide at different doses for control of Meloidogyne spp. in tomato. The experiment was established in the municipality of Tamiahua, Veracruz, using a design randomized complete blocks, with 5 treatments and 4 replications, evaluating the following products: nematicide Vydate L® at doses of 0.75 liters / ha-1, 1.0 liters / ha-1 and 2.0 liters / ha-1, Nemator ${ }^{\circledR}$ nematicide at a dose of $1.0 \mathrm{~kg} /$ ha-1, and an absolute control; 3 applications performing cultivation, these were carried out at the time of transplant, 20 days after transplanting (DAT), at 40 and 60 DAT, respectively. The nematicide Nemator ${ }^{\circledR}$ at a dose of $1.0 \mathrm{~kg} / \mathrm{ha}-1$ controla successfully tomato galling caused by Meloidogyne spp. Vydate L® as a dose of $2.01 /$ ha-1, the latter also presents good control against sucking insects, in the presence of witness aphid Myzus persicae was found, compared to other treatments. Based on the analysis of the results, treatment is recommended because seedling assimilation and improvement exists saving products.
\end{abstract}

Keywords: Nematode, control, tomato.

\section{INTRODUCCIÓN}

El tomate es sin duda la hortaliza más importante a nivel nacional, considerando la superficie sembrada y consecuentemente el valor de la producción. En el aspecto social representa una de las fuentes de empleo más importantes en nuestro país, dado el carácter de mano de obra intensiva que ocupa, en el noroeste de México, la producción de este cultivo para exportación es considerada como una de las más modernas en el ámbito internacional. Esto es, debido a la utilización de tecnología moderna que incluye entre otras cosas; nuevas variedades con larga vida de anaquel y resistencia a algunas enfermedades; así como la utilización de altas densidades de plantas por hectárea, además del uso de fertirrigación con acolchados plásticos para retener por un mayor período la humedad, controlar las malezas y patógenos mediante la fumigación del suelo. Ha sido un factor importante en la producción. Sin embargo estas prácticas han tenido un efecto en el comportamiento de la microflora, microfauna, plantas superiores, y las interrelaciones entre ellas. Ello ha originado alteraciones en las condiciones del suelo, en las que se incluyen la textura, la temperatura, la humedad, la aireación, el $\mathrm{pH}$, la fertilidad y el contenido de materia orgánica. Estos factores han propiciado que las

poblaciones de microorganismos patógenos como el nemátodo agallador de raíces Meloidogyne spp. Se ha reportado en Guanajuato, Michoacán, Guerrero, Oaxaca, Sinaloa, México, Veracruz, Coahuila, Durango, Nuevo León, Tamaulipas, Nayarit, Chiapas, Puebla, Sonora, Tlaxcala y Baja California 
(Sasser, 1977; Montes, 1988; Spooner et al., 2005). Los síntomas del ataque de Meloidogyne spp. Son enanismos de la planta y amarillamiento de las hojas. Las plantas manifiestan síntomas de deficiencia de agua en las horas de mayor calor, aún en presencia de riego adecuado, lo cual se debe a la poca capacidad de la planta atacada para aprovechar el agua disponible. Los síntomas típicos se presentan en el sistema radical donde las plantas atacadas son en general más cortas y sin ramificaciones laterales, y el síntoma más característico es la formación de agallas o tumores. El sistema radical así deteriorado no es capaz de absorber el agua y los nutrientes disponibles en el suelo $\mathrm{y}$, en consecuencia las plantas sufren retardo en el crecimiento (Taylor y Sasser, 1978; Sorribas, Verdejo-Lucas 1999).

En la actualidad se realizan cada vez más aplicaciones de productos químicos encaminados a combatir esta plaga; sin embargo, la aplicación excesiva de plaguicidas afecta de manera negativa de diferentes maneras: la salud humana, la eliminación de la fauna benéfica en los cultivos, selección de organismos resistentes a los plaguicidas, presencia de residuos en frutos y mayor contaminación ambiental (Castellanos, 2009). En base a lo anterior, se considero como objetivo, Evaluar la efectividad biológica de un nematicida a varias dosis para el control de Meloidogyne spp. en tomate (Solanum lycopersicum).

\section{MATERIALES Y MÉTODOS}

El experimento se estableció en un invernadero de producción ubicado en el kilómetro 5.5 de la carretera TuxpanTamiahua, en el municipio de Tamiahua, Veracruz. El clima es cálido-extremoso con lluvias en verano y ocasionalmente en invierno. La precipitación media anual es de 1,500 mm y se concentra en los meses de Julio a Octubre. Temperatura media anual oscila entre los $23{ }^{\circ} \mathrm{C}$, con máximas de hasta $43{ }^{\circ} \mathrm{C}$ en el verano y mínimas de $10{ }^{\circ} \mathrm{C}$ en el invierno. La humedad atmosférica media anual es de $85 \%$, presentándose la máxima, de $89 \%$ en septiembre y la mínima de $51 \%$ en abril (INEGI, 2013). Se utilizó el diseño de bloques completos al azar, con 5 tratamientos y 4 repeticiones, y estuvieron constituidas por 4 camas de $25.0 \mathrm{~m}$ de longitud, con separación entre surcos de 1.8 $\mathrm{m}$. Las parcelas útiles las formaron los 2 surcos centrales, de los que se eliminaron $1.0 \mathrm{~m}$ en cada extremo. El invernadero tiene una superficie de $2,100 \mathrm{~m}$ con cubierta de plástico de calibre de espesor de 600, con ventanas laterales operadas manualmente y ventanas cenitales fijas. Para el desarrollo de tomate se utilizó el propio suelo el cual tiene una textura franco-arenosa, conductividad eléctrica de $1.22 \mathrm{dSm}$ y $\mathrm{pH}$ de 6.96. Los tratamientos evaluados consistieron en diferentes dosis del nematicida Vydate L®, el nematicida Nemator y un testigo absoluto. Como tratamientos que se consideraron para la evaluación de la efectividad biológica son los siguientes: Nematicida Vydate $L \AA$ a dosis de 0.75 lts/ ha ${ }^{-1}, 1.0$ lts/ $\mathrm{ha}^{-1}$ y $2.0 \mathrm{lts} /$ $\mathrm{ha}^{-1}$, el nematicida Nemator a dosis de 1.0 kgs/ $\mathrm{ha}^{-1}$, y un testigo absoluto. Las aplicaciones se llevaron a cabo a través del sistema de riego por goteo con ayuda de una bomba motorizada marca arimitzu. Se realizaron 3 aplicaciones al cultivo, estas se llevaron a cabo al momento del trasplante, a los 20 días después del trasplante, a los 40 días después del trasplante y a los 60 días después del trasplante. El gasto de agua por hectárea fue de 10,000 litros. El hibrido que se utilizó fue el tomate saladette 
indeterminado Ramsés de la casa comercial Harris Moran. El cual posee resistencia al virus rizado amarillo del tomate (TYLCV), virus de la marchitez manchada del tomate. Poseen tolerancia a Fusarium oxysporum spp. lycopersici raza 1,2 y 3 y además poseen tolerancia al nemátodo Meloidogyne incognita en cual se encuentra presente en el suelo del invernadero de producción. Las variables de respuesta utilizadas fueron: Población de nemátodo en el suelo, Efectividad biológica y Fitotoxicidad al cultivo.

\section{RESULTADOS}

presentó el mayor promedio de juveniles, con promedio de 345.5. En el segundo muestreo que fue a los 60 días después del trasplante, se encontraron diferencias estadísticas significativas en la población de nemátodos entre los tratamientos con respecto al testigo, presentando la mayor respuesta el tratamiento Nemator 1.0 $\mathrm{kgs} / \mathrm{ha}^{-1}$, este seguido de Vydate L® a dosis de 2 lts $/ \mathrm{ha}^{-1}$; seguido de Vydate L® a 1.0 y $0.75 \mathrm{lts} / \mathrm{ha}^{-1}$, respectivamente; estos últimos tratamientos interactuaron estadísticamente entre ellos. $\mathrm{El}$ tratamiento testigo fue en donde se registró la mayor concentración de la población de nemátodos juveniles con 396.50 y fue estadísticamente diferente a los demás tratamientos. En el tercer muestreo realizado se encontraron diferencias estadísticas significativas, siendo los mejores tratamientos el Nemator $1.0 \mathrm{kgs} / \mathrm{ha}^{-1}$ y Vydate L® 2.0 lts $/ \mathrm{ha}^{-1}$ con 173.5 y 180.5 juveniles respectivamente, estos tratamientos interactuaron estadísticamente entre sí; seguidos de los tratamientos y Vydate

ParaL®la1población.0y0.75 de1.0nemátodoslts/ha ${ }^{-1}$ quexistentesmostraronen el primer muest promedios de 277.50 y 293.25 juveniles

por $100 \mathrm{~g}$ de suelo respectivamente, estos tratamientos interactuaron estadísticamente entre ellos. Todos los tratamientos fueron estadísticamente diferentes del tratamiento testigo que registró el mayor número de juveniles con 421.35. Figura 1.

\section{Población de Nemátodos}

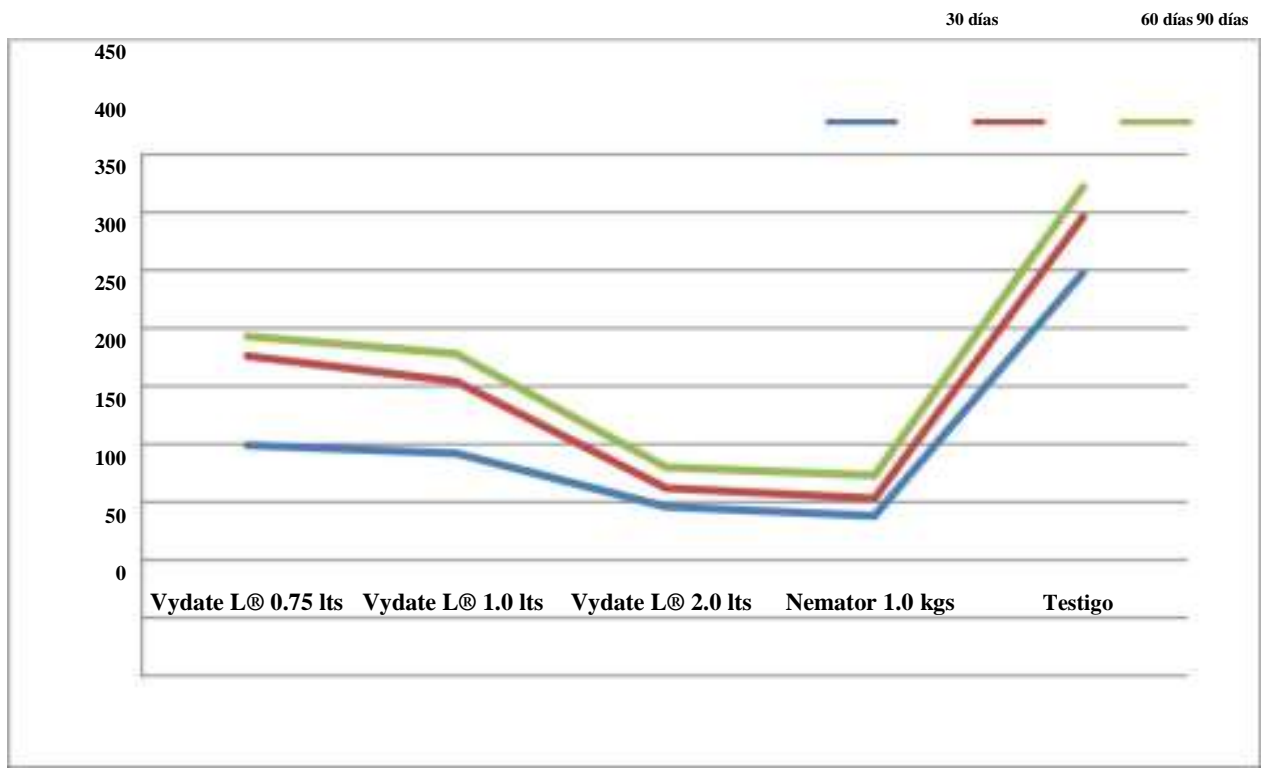

Revista Científica Biológico Agropecuaria Tuxpan 4 (2) 
Figura 1. Población promedio de nemátodos encontrados en el suelo a los 30, 60 y 90 días.

En la variable de respuesta Efectividad Biológica, existieron diferencias estadísticamente significativas entre los tratamientos a los 25 días después de la aplicación. La mayor respuesta de efectividad biológica se registró donde se aplicó dosis de Nemator $1.0 \mathrm{kgs} / \mathrm{ha}^{-1}$ con $2 \%$ de agallamiento, seguido de Vydate L® 2.0 lts $/ \mathrm{ha}^{-1}$ con el $3 \%$ de agallamiento; estos a su vez seguidos de Vydate L 1.0 y 0.75 lts $/ \mathrm{ha}^{-1}$ respectivamente con $5 \%$ y $7 \%$; todos estos tratamientos interactuaron

estadísticamente entre ellos. El tratamiento testigo fue en donde se registró la menor efectividad biológica para el control de nemátodo con el $26 \%$ de agallamiento y fue diferente estadísticamente del resto de los tratamientos. El tratamiento más eficiente y que mantuvo la mejor respuesta positiva a los 25 ddt fue donde se aplicó Nemator $1.0 \mathrm{kgs} / \mathrm{ha}^{-1}$ con el $89.5 \%$ de efectividad, seguido de Vydate L® $2.0 \mathrm{lts} / \mathrm{ha}^{-1}$ con el $86.5 \%$ de eficacia, y ambos tratamientos seguidos de donde se aplicó Vydate L® 1.0 lts ha $^{-1}$ con un $84.2 \%$ de eficacia y de Vydate L® 1.0 lts $/$ ha $^{-1}$ con $77.5 \%$ de efectividad. Estos resultados muestran una tendencia similar a los reportados por Verdejo (2009), quien también encontró que las aplicaciones Vydate L® a razón de $2.0 \mathrm{lts} / \mathrm{ha}^{-1}$ controlan hasta en $85 \%$ la infección del nemátodo en plantas de tomate en las primeras etapas fenológicas del cultivo. Se encontraron diferencias estadísticamente significativas entre los tratamientos a los 50 días después del trasplante (Cuadro 7). La mayor respuesta de efectividad biológica se registró donde se aplicó Nemator $1.0 \mathrm{kgs} / \mathrm{ha}^{-1}$ con el $4 \%$ de agallamiento, seguido de donde se aplicó dosis de Vydate L® 2.0 lts $/ \mathrm{ha}^{-1}$ con el $9 \%$ de agallamiento; seguido de
Vydate L $\quad \begin{array}{lllll}1.0 & \text { y } & 0.75 & \text { lts } / \mathrm{ha}^{-1}\end{array}$ respectivamente con $11 \%$ y $13 \%$; estos tratamientos interactuaron estadísticamente entre ellos. El tratamiento testigo fue en donde se registró la menor efectividad biológica para el control de nemátodo con el 33\% de agallamiento y fue diferente estadísticamente del resto de los tratamientos.

El tratamiento más eficiente y que mantuvo la mejor respuesta positiva a los 50 ddt fue donde se aplicó Nemator 1.0 $\mathrm{kgs} / \mathrm{ha}^{-1}$ con el $90 \%$ de eficacia, seguido de donde se aplicó Vydate L® 2.0 lts $/ \mathrm{ha}^{-1}$ con el $88.5 \%$ de eficacia, ambos seguidos de Vydate L® 1.0 y Vydate $0.75 \mathrm{lts} / \mathrm{ha}^{-1}$ con un $86.3 \%$ y $79.7 \%$ de eficacia respectivamente. Estos resultados muestran una tendencia similar a los reportados por Verdejo (2009), quien también reportó que las aplicaciones Vydate L® a razón de 2.0 lts $/ \mathrm{ha}^{-1}$ a los 60 ddt controlan hasta en $89 \%$ la infección del nemátodo en plantas de tomate.

En este orden de ideas observamos que hubo diferencias estadísticamente significativas entre los tratamientos a los 75 días después del trasplante (Cuadro 8). La mayor respuesta de efectividad biológica se registró donde se aplicó Nemator $1.0 \mathrm{kgs} / \mathrm{ha}^{-1}$ con el $9 \%$ de agallamiento, seguido de donde se aplicó una dosis de Vydate L® 2.0 lts/ha ${ }^{-}$ 1 con el $11 \%$ de agallamiento; ambos tratamientos seguidos de Vydate L 1.0 y $0.75 \mathrm{lts} / \mathrm{ha}^{-1}$ respectivamente con $15 \%$ y $19 \%$; estos dos últimos tratamientos interactuaron estadísticamente entre ellos. El tratamiento testigo fue en donde se registró la menor efectividad biológica para el control de nemátodo con el $65 \%$ 
de agallamiento y fue diferente estadísticamente del resto de los tratamientos. El tratamiento más eficiente y que mantuvo la mejor respuesta positiva a los 75 ddt fue donde se aplicó Nemator $1.0 \mathrm{kgs} / \mathrm{ha}^{-1}$ con el $84 \%$ de eficacia, seguido de Vydate L® $2.0 \mathrm{lts} / \mathrm{ha}^{-1}$ con el $80 \%$ de eficacia, ambos tratamientos seguidos de donde se aplicó Vydate L® 1.0 y $0.75 \mathrm{lts} / \mathrm{ha}^{-1}$ con un $77 \%$ y $71 \%$ de eficacia respectivamente. Estos resultados muestran una tendencia similar a los reportados por Verdejo (2009), quien también reportó que las aplicaciones Vydate L® a razón de 2.0 lts/ha ${ }^{-1}$ sigue manteniendo una efectividad superior al $80 \%$ para el control de la infección del nemátodo en plantas de tomate.

\section{CONCLUSIONES}

Con los resultados obtenidos se puede concluir lo siguiente: El Nematicida Nemator a dosis de 1-0 kilogramos por hectárea, controla satisfactoriamente el agallamiento del tomate causado por Meloidogyne spp. $Y$ su resultado es estadísticamente igual a la aplicación de Vydate L® a dosis de 2.0 litros por hectárea. El nematicida Vydate L® a dosis de $0.75,1.0$ y 2.0 litros por hectárea, controla satisfactoriamente el agallamiento del tomate causado por Meloidogyne spp.. Aplicaciones del nematicida Vydate $L \circledR$ a dosis de 0.75 , 1.0 y 2.0 litros por hectárea, reducen eficazmente las poblaciones de nemátodos en el suelo y el porcentaje de agallamiento del tomate. La dosis del nematicida Vydate $L \circledR$ a dosis de 0.75 , 1.0 y 2.0 litros por hectárea para controlar al nemátodo Meloidogyne spp., causante del agallamiento en tomate es de 0.75 a 2.0 litros por hectárea. El nematicida
Vydate L® a dosis de $0.75,1.0$ y 2.0 litros por hectárea, no causa problemas de Fitotoxicidad al cultivo de tomate. El nematicida Vydate L® es una buena alternativa para el manejo del agallamiento del tomate, causado por Meloidogyne spp. Además de controlar efectivamente al nemátodo agallador, el nematicida Vydate $L \circledast$, presenta buen control contra insectos chupadores, ya que en el testigo se encontró presencia de pulgón Myzus persicae, comparado con el resto de los tratamientos.

\section{LITERATURA CITADA}

Carrillo Fasio J. A., García Estrada R. S., Allende Molar R., Márquez Zequera I., Cruz Ortega J. E. 2000. Identificación y Distribución de Especies del Nematodo Nodulador (Meloidogyne spp.) en Hortalizas, en Sinaloa. Revista Mexicana de Fitopatología, Sociedad Mexicana de Fitopatología, A.C,.vol. 18, núm. 2, pp. 115- 119 .

Castro, A. A.E., E. Zavaleta-Mejia., I. Cid del Prado y V. Zamudio G. 1990. "Rotación e incorporación de Tagetes erecta L. para el manejo de Meloidogyne incognita (Kofoid \& white) Chitwood en el cultivo de tomate (Lycopersicon esculentum Mill.) en Tecamachalco

Puebla. Revista mexicana de Fitopatología 8: 173-180 pp.

De León L., Banchero L., López-Pérez J. A., Bello A. 2000. "Control de Meloidogyne incognita en cultivo de tomate en Uruguay". 7 pp.

Fayos-Albelda, J.M. Camarena, Girbés R. 2013. Estudio de la eficacia de Nematocid contra Meloidogyne spp en Solanum 
lycopersicum (tomate) en España. ENSAYO 13 A TO FI. 10-26 pp.

Hartman, K.M. and J.N. Sasser. 1985. Identification of Meloidogyne species on the basis of differential host tests and perineal pattern morphology. 6977 pp. In: Baker, K.R., C.C. Carter and J.N. Sasser (Eds). An advanced treatise on Meloidogyne. Volume II. Methodology. North Caroline State University Graphis. Raleigh, North Carolina.

Hernández-Martínez， García-Mata R., Valdivia-Alcalá R. y Omaña-Silvestre J. M. 2004. Evolución de la competitividad y rentabilidad del cultivo del tomate rojo (Lycopersicon esculentum L.) en Sinaloa,

México. Especialidad en Economía. Instituto de Socioeconomía, Estadística e Informática. Colegio de Postgraduados.

56230. Montecillo, México. (rory@colpos.mx). División de Ciencias Económico Administrativas. Universidad

Autónoma Chapingo. Chapingo. México.111 pp.

Leyva Pérez A. R., Castellanos González L., Pérez Fernández. 2011. "Alternativas de lucha contra nemátodos noduladores en el cultivo del tomate en condiciones de organopónicos".

López-Linares I. G., 2009. "Control de Meloidogyne sp., en viveros de café (Coffea arabica L.), mediante el hongo Paecilomyces lilacinus". Universidad de el salvador facultad de ciencias agronómicas departamento de protección vegetal. $88 \mathrm{pp}$.

Perichi G. y Crozzoli R. 2010. “Morfología, Morfométría y hospedantes diferenciales de poblaciones de Meloidogyne de los estados Aragua y
Zulia, Venezuela". Universidad Central de Venezuela, Facultad de Agronomia.

Instituto de Zoología Agrícola, Laboratorio de Nematología. Agrícola, Apartado Postal 4579, Maracay 2101-A, estado Aragua, Venezuela.

Suárez Hernández, Z.M. 1989. Respuesta de 10 variedades de tabaco al ataque de Meloidogyne incognita raza 1 y Meloidogyne javanica, inoculadas en forma individual y combinada. Tesis de Maestría. Colegio de Posgraduados, Montecillo Estado de México. 72 pp.

Taylor, A.L. and J.N. Sasser. 1978. Biology, identification and control of

root-knot nematodes (Meloidogyne species). Raleith, Nort Caroline State University Graphics. 111 pp.

Terry E., Ruiz J. 2008. Evaluación de bioproductos para la producción de tomate (Solanum Lycopersicum, Mill) bajo sistema de cultivo protegido Cultivos Tropicales, Instituto Nacional de Ciencias Agrícolas Cuba, vol. 29, núm. 3, pp. 11-15. 
Copyright (c) 2016 Victor Manuel Figueroa Hernández,

Brenda Mariana Hernández Mar y Mario Alberto Rom án Martinez

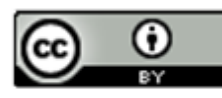

Este tex to está protegi do por una licencia licencia Creative Commons 4.0.

Usted es libre para Compartir —copiar y redistribuir el material en cualquier medio o form ato- y Adaptar el documento —remezdar, transformar y crear a partir del material- para cualquier propósito,, incluso para fines comerciales, siempre que cumpla la condición de:

Atribución: Usted debe dar crédito a la obra original de manera adecuada, proporcionar un enlace a la licencia, e indicar si se han realizado cam bios. Puede hacerlo en cualquier form a razonable, pero no de form a tal que sugiera que tiene el apoyo del licenciante o 10 recibe por el uso que hace de la obra.

Resumendelicencia - Textocompletodelalicencia 